\title{
NANOTECNOLOGIA: SIMULAÇÃO DE SUPERFÍCIES HIDROFÓBICAS POR MEIO DE MATERIAIS ALTERNATIVOS
}

\section{NANOTECHNOLOGY: SIMULATION OF HYDROFOVIC SURFACES BY ALTERNATIVE MATERIALS}

Flávio José de Abreu Moura ${ }^{1}$; Palloma Joyce de Aguiar Silva ${ }^{2}$; Eduardo Fragôso dos Santos $\mathrm{Silva}^{3}$; Fernando Cleyton Henrique de Mendonça Silva ${ }^{4}$; Etelino José Monteiro Vera Cruz Feijo de Melo $^{5}$

\section{RESUMO}

As superfícies hidrofóbicas têm despertado um grande interesse em variadas áreas devido à sua importância e a suas potenciais aplicações industriais. As moléculas hidrofóbicas normalmente são apolares, portanto não há atração entre as moléculas de água que são polares. Esses materiais que não possuem uma boa interação com a água já são comercializados mundialmente: camisas que não ficam sujas, sapatos que não permitem a passagem de líquidos, vidros de carros que facilitam o deslize da água tendo uma melhor visualização em dias chuvosos e até mesmo na medicina com microdispositivos que atacam exatamente o local desejado. A molhabilidade da superfície de um material é uma propriedade que está presente também no cotidiano, e muitas vezes não são facilmente visualizadas, estando presente em diversas plantas, penas das aves e até mesmo nas asas de alguns insetos. Dessa forma considera-se que a Nanotecnologia está presente no cotidiano da população e é indispensável que esse assunto seja levado para as aulas de Química do ensino médio. De acordo com alguns estudos compete ao ensino médio o papel essencial na formação do cidadão através de temas relevantes e atuais como a Nanociência e a Nanotecnologia, além de outros de forte relação com aspectos sociais e ambientais. Por esse motivo apresentamos esse trabalho, no qual mostramos alguns materiais que podem ser utilizados para simular algumas práticas Nanotecnológicas voltadas para o ensino. A pesquisa foi realizada com professores do curso de Licenciatura em Química e integrantes do GT de experimentação do PDVL. Foi possível observar que os materiais realmente simulam superfícies hidrofóbicas e são de fácil acesso podendo ser utilizados por professores como ferramenta auxiliadora no ensino de Química.

Palavras-Chave: Materiais Hidrofóbicos, Nanotecnologia, Ensino Médio, Ensino de Química.

\footnotetext{
ABSTRACT

Hydrophobic surfaces have aroused a great deal of interest in various areas because of their importance and potential industrial applications. Hydrophobic molecules are usually nonpolar, so there is no attraction between water molecules that are polar. These materials that do not have a good interaction with the water are already sold worldwide: shirts that do not get dirty,

${ }^{1}$ Licenciatura em Química, Instituto Federal de Educação Ciência e Tecnologia de Pernambuco - Campus Vitória, flavio.jose33@ hotmail.com

${ }^{2}$ Licenciatura em Química, Instituto Federal de Educação Ciência e Tecnologia de Pernambuco - Campus Vitória, palloma_joyce_aguiar@hotmail.com

${ }^{3}$ Licenciatura em Química, Instituto Federal de Educação Ciência e Tecnologia de Pernambuco - Campus Vitória, eduardofragoso06@gmail.com

${ }^{4}$ Mestrando em Educação de Ciências e Matemática, Universidade Federal de Pernambuco - Centro Acadêmico do Agreste, silva.fchm@gmail.com

${ }^{5}$ Doutor em Química, IFPE-campus Vitória de santo antão, etelino.melo@vitoria.ifpe.edu.br
} 
shoes that do not allow the passage of liquids, glasses of cars that facilitate the water slide having a better visualization on rainy days and even in medicine with microdevices that attack exactly the desired location. The wettability of the surface of a material is a property that is also present in everyday life, and is often not easily visualized, being present in several plants, bird feathers and even the wings of some insects. In this way it is considered that Nanotechnology is present in the daily life of the population and it is indispensable that this subject be taken to the classes of Chemistry of high school. According to some studies, it is the responsibility of secondary education to play an essential role in the formation of the citizen through relevant and current topics such as Nanoscience and Nanotechnology, as well as others with a strong relationship with social and environmental aspects. For this reason we present this work, in which we show some materials that can be used to simulate some nanotechnology practices focused on teaching. The research was carried out with professors of the Licentiate course in Chemistry and members of the PDVL experimentation GT. It was possible to observe that the materials actually simulate hydrophobic surfaces and are easily accessible and can be used by teachers as a help tool in the teaching of Chemistry.

that contribute to the students' participation in the class. Thus, the study has the objective of analyzing the role of the didactic game Periodic Race in the appropriation of the symbology characteristic of the content of Periodic Table. This is a qualitative research carried out in two classes in grade 10 at a Secondary School in Salgadinho-PE. The game was proposed by the researchers and applied by the class teacher. We used as instruments the researcher's field diary and interviews with some students. In the analysis of the data produced, we show the interactions between the teacher and the students in the game and the points of view of the students in the interview. In the course of the game we noticed that the students knew only the most commonly seen chemical elements in their daily life and they presented difficulties in the periodic properties, besides not knowing the importance of the symbology of this content. With the game, the students were able to have direct contact with the Periodic Table, knowing it properly and slowly demystifying the language present in it, understanding that it brings a knowledge based in studies and that effectively contributes to various chemistry contents. Therefore, the use of activities such as the didactic game provides an environment conducive to this appropriation of the scientific school language in chemistry, as well as learning the meanings that demystify the contents' chemistry language.

Keywords: Hydrophobic Materials, Nanotechnology, High School, Chemistry Teaching

\section{INTRODUÇÃO}

A manipulação de materiais nanométricos tem avançado em seus estudos nos últimos anos. Ressalta-se que esses materiais apresentam propriedades em diversas áreas do conhecimento, como a medicina, os fotovoltaicos, entre outros.

Além disso, esse assunto tem sido abordado como tema em concursos, tal como o Exame Nacional do Ensino Médio - ENEM, o qual seleciona os alunos do ensino médio para ingressar no ensino superior. Nesse sentido, os conteúdos que envolvem a nanotecnologia podem ser facilmente abordados como proposta interdisciplinar e complementar pelos professores de Química do ensino médio.

Assim, salientamos que a inserção de temáticas atuais no ensino de ciências, de forma 
que os alunos percebam as modificações e avanços científicos recentes, é de fundamental importância, para que esse amadurecimento possa ocorrer gradativamente a partir da apreensão de saberes e conhecimentos já consolidados e os que se encontram em desenvolvimento.

Com a utilização da experimentação no ensino de Química há uma maior possibilidade de aprendizado, pois abrange uma nova perspectiva de ensino, tornando o aluno um ser pensante, criativo e construtor do conhecimento.

Diante de tal importância, o Programa Internacional Despertando Vocações para Licenciaturas - PDVL tem um Grupo de Trabalho de Experimentação. Dentre as ações de experimentações está a área de Nanotecnologia. Mas é preciso saber o que pensam os professores que ministram Química no Ensino Médio. Afinal, o tema de nanotecnologia já está presente até no ENEM. Surge então uma preocupação, segundo (VIANA, 2014) os professores que ministram a disciplina de Química no Ensino Médio, em sua maioria, não têm formação na área.

Dessa forma o objetivo do presente trabalho foi analisar as condições necessárias de ordem estrutural e conceitual para a construção de materiais alternativos que possibilitassem praticas experimentais em nanotecnologia simulando superfícies hidrofóbicas para serem utilizados em salas de aula ou laboratórios de Química.

Utilizamos como recursos didáticos, uma apresentação em powerpoint para apresentar o problema e a explanação do conteúdo, e também, práticas experimentais, nas quais um dos experimentos simulava o que o problema estava tratando.

\section{FUNDAMENTAÇÃO TEÓRICA}

Nanotecnologia é a habilidade de manipular átomos e moléculas individualmente para produzir materiais nanoestruturados e micro objetos com aplicações no mundo real (Miller, 2005). A tecnologia nano já é um negócio de bilhões de dólares (Bushan, 2004) que atrai novos investimentos a cada dia em todo o planeta, devido ao seu enorme potencial de aplicação nos mais variados setores industriais e ao impacto que seus resultados podem dar ao desenvolvimento tecnológico e econômico.

A Nanociência trabalha com a escala da bilionésima parte do metro, correspondendo ao nanômetro. Já a Nanotecnologia faz uso dessas possibilidades e gera produtos utilizáveis e funcionais. Alguns desses produtos já eram desenvolvidos mesmo antes da adoção formal do termo Nanociência, de forma mais rústica, como os vitrais coloridos em igrejas medievais, os quais eram obtidos utilizando partículas de ouro e outros minerais, em tamanhos 
diferenciados, incluindo a escala nano, para apresentar cores diferentes quando misturados com o vidro. Atualmente esses e muitos outros produtos são sintetizados em laboratórios, o que permite suas aplicações em larga escala (SCHULZ, 2006).

Segundo Zanella et al. (2009), a nanotecnologia promete revolucionar a forma como o ser humano vive, se comunica e trabalha. Devido a esse grande avanço, é importante que as instituições de ensino fundamental e médio abordem a nanotecnologia em suas atividades escolares, visto que a probabilidade de os mesmos se inserirem no mercado de trabalho e lidarem com a $\mathrm{N} \& \mathrm{~N}$ (Nanotecnologia e Nanociência) tem se tornado cada vez mais crescente.

Roco (2003), afirma que os conceitos em escala atômica, molecular e níveis supramoleculares devem ser inseridos no sistema educacional da atualidade, pois dentre os principais desafios para o desenvolvimento da nanotecnologia encontra-se a educação e formação de uma nova geração de trabalhadores qualificados na perspectiva multidisciplinar.

Há necessidade de alfabetizar cientificamente nossos estudantes, para que estes compreendam o mundo tecnológico que os cercam, possibilitando entender o funcionamento de alguns equipamentos que fazem parte do seu dia a dia. Porém, muitas vezes os alunos não possuem informações suficientes para tal entendimento (HEALY, 2009), o que pode ser atenuado com a organização de materiais didáticos atualizados, inclusive entre eles os que se referem às Nanociências.

Precisamos de conteúdos relacionados às Nanociências que sejam diferenciados e de fácil entendimento aos usuários, que expliquem minimamente estas novas aplicações. As perspectivas para o futuro, nesta área, são as mais diversas, como a possibilidade de aumento da memória e velocidade dos computadores, cabos feitos de nano tubos e nano fios de carbono, novos materiais inteligentes, construção de nano máquinas, as quais poderão inserir medicamentos no corpo humano e em locais específicos (BARONE, 2005).

\section{METODOLOGIA}

\section{Caracterização da pesquisa}

A pesquisa foi de natureza qualitativa, onde foi realizada uma análise de ordem estrutural e conceitual das condições necessárias para a construção de materiais alternativos para aulas experimentais de química. O campo de pesquisa foi o IFPE - Campus Vitória de Santo Antão e o Programa Internacional Despertando Vocações para Licenciaturas (PDVL), no ano de 2018. Os sujeitos foram professores da Licenciatura em Química e alunos integrantes do Grupo de Trabalho de Experimentação. 


\section{Procedimentos metodológicos}

Inicialmente foram analisados os planos de ação do PDVL com relação às práticas experimentais. Em seguida foi realizado entrevistas semiestruturadas com professores do curso de Licenciatura em Química e licenciandos participantes do GT de Experimentação: P1: "Você acha importante falar sobre Nanotecnologia no ensino médio?", P2: "Você acha que é possível realizar praticas experimentais no ensino médio que de fato utilize manipulação atômica?", P3: “Quais materiais podem ser utilizados para representar matérias hidrofóbicos?"

Também foram realizadas observações, registros, análise da construção de materiais para práticas experimentais em nanotecnologia.

\section{RESULTADOS E DISCUSSÃO}

\section{- Análise dos planos de ação do PDVL em relação às práticas experimentais:}

Para iniciarmos nossa pesquisa, foi de suma importância analisarmos os documentos orientadores do PDVL, afinal, foram pesquisas realizadas com integrantes deste Programa. Assim, observamos que o PDVL é um programa que iniciou seu planejamento no dia 19 de novembro de 2013 e suas ações em 01 de fevereiro de 2014. Hoje, o Programa conta com um número maior de instituições parceiras nacionais: IFPE (Instituto Federal de Pernambuco), IFPB (InstitutoFederaldaParaíba), IFAL (InstitutoFederaldeAlagoas), IFRO(InstitutoFederal de Rondônia), IF-SE PE (Instituto Federal do Sertão Pernambucano), IFPI (Instituto Federal do Piauí), FACHUSC (Faculdade de Ciências Humanas - Salgueiro). O programa também conta com as seguintes instituições parceiras internacionais: UM (Universidad de Mendonza Argentina), UNLP (Universidad Nacional de La Plata - Argentina), UTN (Universidad Tecnológica Nacional de Mendoza - Argentina), UPLA (Universidad de Playa Ancha Chile), MEDUCA (Ministério da Educação - Panamá). Onde se divide nas seguintes áreas: Química, Física, Matemática, Geografia, Biologia, História, Letras, Computação.

Todas as ações do PDVL do IFPE - campus Vitória são na área de Química, dos bolsistas e voluntários são licenciandos da área e as ações são desenvolvidas a partir de GT (Grupos de Trabalhos), a saber: GT de Jogos Didáticos, GT de Experimentação, GT de Avaliação- Resolução de Problemas, GT de Softwares Educativos, GT de Tecnologias Educacionais, Laboratório de Avaliação, que agrega todos os GT’s, como espaço de testagem 
e validação das ações antes de ir para as escolas. Ressaltamos que além das ações nas escolas ainda são realizadas Visitas Guiadas, onde são trazidos estudantes das escolas para visitarem o IFPE e além das ações online, com oferecimento de cursos na plataforma pdvlearning para licenciandos e professores.

Com relação ao GT de Experimentação, observamos que os extensionistas se reúnem para planejamento das ações em reuniões semanais com duração de 3 horas. Nesses encontros ocorrem estudos sobre abordagens experimentais e conteúdo específicos da química. Todos os assuntos abordados nos experimentos são baseados no levantamento inicial que fazem nas escolas parceiras do programa.

Assim, no início de cada ano, os extensionistas realizam um diagnóstico, através de um questionário com perguntas fechadas, sobre a carreira docente em química e as principais dificuldades na disciplina de Química. Entre as perguntas, são elencados assuntos para que os estudantes do Ensino Médio possam identificar aqueles que têm mais dificuldade de aprendizagem.

- Entrevista com professores do Ensino Superior e dos Licenciandos participantes do GT de Experimentação PDVL

A análise dos resultados obtidos na $\mathbf{P 1}$ da entrevista proporcionou evidenciar que $100 \%$ dos licenciandos e os professores consideram importante falar sobre nanotecnologia no ensino médio. Eles alegaram que “...trazer essa realidade para os alunos logo cedo é uma boa forma de mostrar o impacto da tecnologia no dia-a-dia...", "pois existem várias tecnologias no nosso cotidiano que utiliza da nanotecnologia como ferramenta de progresso" como "...por exemplo: cosméticos, televisões, tratamento de câncer e outros."

Quando os entrevistados foram questionados se era possível realizar práticas de manipulação atômica no ensino médio $\mathbf{P 2}, 82 \%$ afirmaram que não era possível porque era necessário equipamentos muito sofisticados e era inviável trazê-los para uma sala de aula no ensino médio.

Em seguida perguntamos alguns matérias que poderiam ser utilizados para simular algumas superfícies hidrofóbicas já que a utilização dos equipamentos adequados era inviável. Os professores e alunos deram algumas sugestões e todas eles se resumem a materiais que sejam apolares visto que a água possuí polaridade, logo, eles irão se repelir. 
- Analise para a construção de materiais para praticas experimentais em nanotecnologia no ensino médio

Após pesquisar bastante e conversar com professores de Química observamos que o melhor a se fazer seria utilizar experimentos representativos, que simulassem algumas práticas nanotecnológicas. Dessa forma separamos alguns experimentos que simulam materiais hidrofóbicos e de baixo custo, que podem ser realizados pelos estudantes:

\begin{tabular}{|c|l|}
\hline $\begin{array}{c}\text { Tinta Cristalina com desodorante } \\
\text { aerossol }\end{array}$ & $\begin{array}{l}\text { Primeiro coloca a tinta sobre a superfície do } \\
\text { vidro e logo em seguida pressiona o aerosol } \\
\text { por cima da camada de tinta. Um composto } \\
\text { presente no aerosol proporciona que o vidro } \\
\text { fique super hidrofóbico, impedindo que a } \\
\text { água tenha uma grande superfície de contato. }\end{array}$ \\
\hline Raspa de vela & $\begin{array}{l}\text { Esse experimento é bem conhecido, consiste } \\
\text { em raspar vela em tecidos, como se estivesse } \\
\text { pintando. A vela é composta por parafina } \\
\text { (Hidrocarbonetos), todo hidrocarboneto não } \\
\text { possui polaridade, e como a água é polar eles } \\
\text { não se misturam dando um aspecto } \\
\text { hidrofóbico. }\end{array}$ \\
\hline Fuligem & $\begin{array}{l}\text { Utilizando uma vela ou qualquer outra fonte } \\
\text { de chama, podemos adquirir a fuligem. A } \\
\text { fuligem ocorre quando não há uma } \\
\text { combustão completa formando uma } \\
\text { superfície escura característica. Esse material } \\
\text { possui uma superfície rugosa diminuindo a } \\
\text { superfície de contato das gotas de água, } \\
\text { tornando um material hidrofóbico. }\end{array}$ \\
\hline Folhas da flor de lótus & $\begin{array}{l}\text { As folhas da flor de lótus são inspirações para } \\
\text { pesquisas acerca de materiais hidrofóbicos, é } \\
\text { um ótimo exemplo presente na natureza. Suas } \\
\text { folhas são formadas por uma rugosidade que } \\
\text { diminui a superfície de contato das gotas de } \\
\text { água. Essa rugosidade permite que suas } \\
\text { folhas se mantenham sempre limpas. }\end{array}$ \\
\hline
\end{tabular}

\section{CONCLUSÕES}

Diante de tudo isso observamos que o PDVL é um programa grandioso, que tem uma abrangência ampla na América Latina e que muitos parceiros desenvolvem as ações tanto no Brasil, quanto em outros países.

Após analisado as concepções de alunos e professores do campus - IFPE Vitória de 
Santo Antão a respeito da Nanotecnologia é perceptível que há uma carência muito grande na inserção pertinente e relevante do assunto. Como os alunos entrevistados são de diferentes cidades abrangendo boa parte do estado pernambucano, é perceptível que esse não seja um problema situado apenas em Vitória de Santo Antão. Como os próprios alunos alegaram, os professores estão cada vez mais preocupados em simplesmente repassar o conteúdo deixando de lado esses contextos que podem facilitar o entendimento dos alunos.

Esses assuntos proporcionam que os estudantes tenham um conhecimento abrangente dos avanços tecnológicos e científicos aproximando cada vez mais da realidade do dia-a-dia. Assuntos como estes são de suma importância para quebrar o paradigma que a química é de difícil compreensão e chata para boa parte dos alunos. Essa contextualização, muitas vezes, pode ser um problema para professores das escolas pela pouca carga horária da disciplina de Química. Já que a contextualização exige um pouco de tempo e preparação do profissional.

Também concluímos que os matérias citados são de fácil acesso e pode ser utilizado facilmente pelos professores de química. Esses instrumentos aqui citados devem ser utilizados para simular resultados que a manipulação atômica nos traz, como por exemplo camisas que não se sujam, sapatos impermeáveis, vidros de carros que tem baixa molhabilidade entre outros.

\section{REFERÊNCIAS}

BRASIL. Parâmetros Curriculares Nacionais - Ensino Médio. 2000. Disponível em: <http://portal.mec.gov.br/seb/arquivos/pdf/14_24.pdf> Acesso em: 15 abr. 2014

BRASIL. Diretrizes Curriculares Nacionais para Educação Básica. 2013. Disponível em: $<$ http://portal.mec.gov.br/index.php?option=com_content\&view=article\&id=12663\&Itemid= 1152> Acesso em: 15 abr. 2014.

BARONE, P. W. et al. Near-infrared optical sensors based on single-walled carbon nanotubes. Nature Materials, v. 4, p. 86-92, 2005.

BUSHAN, B. (Editor), "Springer Handbook of Nanotechnology", First Edition, New York, Springer Verlag, 2004.

NEM. Caderno de Provas. Disponível em: http://www.enem2015.net.br/download-doscadernos-deprovas-do-enem-2015.html. 2015. Acesso em 27 de novembro de 2018.

HEALY, N. Why Nano Education? Journal of Nano Education, v.1, p. 6-7, 2009

MILLER, JOHN C., SERRATO, R., KUNDAHL, G., "The Handbook of Nanotechnology: Business, Policy and Intellectual Property Law", First Edition, New Jersey, Wiley, 2005.

ROCO, M.C. Converging science and technology at the nanoescale: opportunities for 
education and training. Focus on nanobiotechnology careers and recruitment. Nature Biotechnology, v. 21, n. 10, oct., 2003. Disponível em: http://www.nature.com/ nbt/journal/v21/n10/pdf/nbt1003-1247.pdf. Acesso em: 08/04/2014.

SCHULZ, P. A. B. O que é Nanociência e para que serve a Nanotecnologia?.Física na Escola, v. 6, n. 1, p. 58-62, 2006.

ZANELLA, I. et al. Abordagens em nanociência e nanotecnologia para o ensino médio. XVIII Simpósio Nacional de Ensino de Física, 1-9, 2009. 